
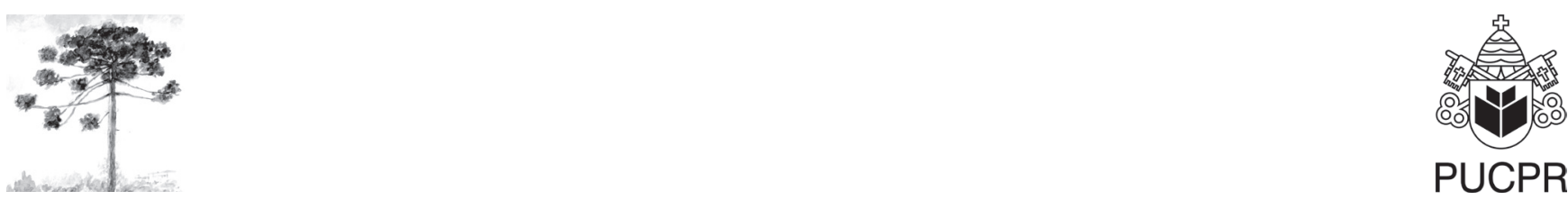

PUCPR

\title{
ANÁLISE PRELIMINAR DA VARIAÇÃO DO LACTATO SANGUÍNEO EM LUTADORES DE MUAY THAI
}

\author{
Preliminary analysis of blood lactate variation in muay thai fighters
}

\author{
Marina de Simas Zachow ${ }^{[a]}$, Fabien Pereira da Silva ${ }^{[b]}$, \\ Selene Elifio Espósito ${ }^{[c]}$, Luiz Fernando Pereira ${ }^{[\mathrm{d}]}$
}

[a] Bióloga, Laboratório de Fisiologia da Pontifícia Universidade Católica do Paraná (PUCPR), Curitiba- PR - Brasil, e-mail: marinazachow@hotmail.com

[b] Profissional de Educação Física, Laboratório de Fisiologia da Pontifícia Universidade Católica do Paraná (PUCPR), Curitiba- PR Brasil, e-mail: fabiensilva@hotmail.com

[c] Professora Doutora, Laboratório de Fisiologia (CCBS), Pontifícia Universidade Católica do Paraná (PUCPR), Curitiba- PR Brasil, e-mail: selene.elifio@pucpr.br

[d] Professor Doutor, Laboratório de Fisiologia (CCBS), Pontifícia Universidade Católica do Paraná (PUCPR), Curitiba- PR - Brasil, e-mail: fernando.pereira@pucpr.br

\section{Resumo}

Este estudo verificou o nível de lactato sanguíneo e a frequência cardíaca (FC) em um grupo de atletas de muay thai. Durante cinco meses coletou-se, de seis lutadores (idade: $26 \pm 3,7$ anos, peso: $76 \pm 12,3 \mathrm{~kg}$, estatura: $170 \pm 9 \mathrm{~cm}$ ), $25 \mu \mathrm{L}$ de sangue para avaliar o nível de lactato (repouso, 0, 1, 3, 5 e $7 \mathrm{~min}$ ), concomitantemente também foi avaliada a frequência cardíaca (Polar - S120). Todo procedimento ocorreu em 5 min no limite máximo de esforço. Os dados experimentais para o lactato foram: (repouso) 2,41 mM, (0) 5,34 mM, (1) 6,62 $\mathrm{mM}$, (3) 6,59 mM, (5) 6,72 $\mathrm{mM}$ e (7) 5,77 mM. Para FC foram: (repouso) 65,48 bat/min, (0) 173,89 bat/ min, (1) 114,07 bat/min, (3) 97,66 bat/min, (5) 95,02 bat/min e (7) 93,1 bat/min. Sendo assim, infere-se que estes dados podem fornecer subsídios para reavaliações de protocolos de treinamento do muay thai, para que os atletas atinjam, no período de competição, um nível de recuperação lática mais efetiva. Com relação à FC, os valores parecem mostrar uma adaptação ao treinamento proposto.

Palavras-chave: Limiar anaeróbio. Frequência cardíaca. Esporte de contato. Lutas marciais.

\section{Abstract}

This study verified the serum lactate level and the cardiac frequency (CF) in a muay thai athletes group. During five months it was collected, in six fighters (age: $26 \pm 3,7$ years, weight: $76 \pm 12,3 \mathrm{~kg}$, 
stature: $170 \pm 9 \mathrm{~cm}$ ), $25 \mu \mathrm{L}$ of blood (ear lobe), to evaluate the lactate level (rest, 0, 1, 3, 5, $7 \mathrm{~min}$ ), concomitantly the cardiac frequency was evaluated (Polar-S120). All the procedures occurred in the maximum limit of effort during $5 \mathrm{~min}$. The experimental lactate results were: (rest) $2.41 \mathrm{mM}$, (0) $5.34 \mathrm{mM}$, (1) $6.62 \mathrm{mM}$, (3) $6.59 \mathrm{mM}$, (5) $6.72 \mathrm{mM} \mathrm{e} \mathrm{(7)} 5.77 \mathrm{mM}$. For CF they had been: (rest) $65.48 \mathrm{bat} / \mathrm{min}$, (0) $173.89 \mathrm{bat} / \mathrm{min}$, (1) $114.07 \mathrm{bat} / \mathrm{min}$, (3) $97.66 \mathrm{bat} / \mathrm{min}$, (5) $95.02 \mathrm{bat} / \mathrm{min} \mathrm{e} \mathrm{(7)} 93.1$ $\mathrm{bat} / \mathrm{min}$. Being thus, it is inferred that these data can supply to subsidies reevaluations of protocols of training for athlete of muay thai so that reach, during competition period, more effective level of lactic recovery. In this regards CF values seems to show an adaptation for the training considered.

Keywords: Threshold lactate. Cardiac frequency. Sports contact. Martial arts.

\section{INTRODUÇÃO}

As artes marciais no geral originaram-se com o intuito de proteção e defesa pessoal, além de sempre ser seguida de certa religiosidade. Mas atualmente são praticadas como esportes, cujo intuito principal é manter a saúde e a boa forma física. Dentre esses esportes de contato, o muay thai, de origem tailandesa, tem-se difundido no Brasil em larga escala.

Para a obtenção do condicionamento físico a um determinado esporte, ou luta, é necessário aprimorar a capacidade aeróbia, além de trabalhar músculos ou sistemas orgânicos específicos com maior intensidade. Segundo Rosa et al. (1), é necessário investir em pesquisas que determinem maior especificidade do treinamento, ou seja, trabalhando grupos musculares ou órgãos específicos, o rendimento do atleta aumenta significantemente $(2,3,4,5)$. Alguns autores acrescentam que um teste deve ser semelhante ao esporte específico do indivíduo, utilizando, na avaliação, a maior massa muscular envolvida durante a performance atlética, aumentando a confiabilidade e o rendimento do atleta ao realizar o protocolo $(6,7)$.

De acordo com Silva et al. (8), o nível de lactato no sangue tem sido um parâmetro metabólico frequentemente utilizado para quantificar a intensidade de esforço realizado pelo atleta durante treinamentos e competições em diversas modalidades esportivas. Apesar de existirem controvérsias na metodologia, o resultado verificado, se bem interpretado, pode trazer informações importantes na orientação do tipo de treinamento e planejamento da dieta a ser seguida pelo atleta $(8,9,10,11)$. Lima et al. (12) relatam que o acúmulo de elevadas concentrações de lactato no sangue durante uma luta pode interferir no desempenho, já que o sucesso na competição também depende de uma rápida remoção de lactato. Se capacidade anaeróbica for insuficiente para manutenção do desempenho, é provável que diminuam as chances de executar uma esquiva ou de contragolpear o adversário (13).

A concentração sanguínea do lactato é modificada pela atividade física, que depende da intensidade, da carga de trabalho e da duração desta $(9,14)$. Em função do aumento da capacidade oxidativa, observada com o treinamento de endurance, acredita-se que esse é capaz de aumentar a habilidade do músculo esquelético de remover o lactato do sangue (15). Autores sugerem que esse metabolismo é dinâmico, pois demonstram que o lactato produzido é rapidamente removido do sangue, mas sua concentração permanece constante, o que indica uma liberação contínua. Além disso, o nível de produção parece não se modificar de indivíduos não treinados para os treinados, sendo que o treinamento físico parece aumentar a captação e não alterar a produção $(11,16,17)$.

É muito importante entender que os benefícios do treinamento são aprimorados quando os programas são planejados de forma a atender as necessidades individuais e as capacidades dos participantes (18). Estudos sugerem que a prática de exercícios físico, em intensidades até o limiar lático, parece proporcionar ao praticante maior segurança em relação aos riscos cardiovasculares (19). Relata-se ainda que a sobrecarga do sistema anaeróbico possa melhorar o desempenho, e para se observar essa alteração é possível coletar o lactato sanguíneo correlacionando-o com a frequência cardíaca (13).

Baseado nas informações anteriores, este trabalho avaliou o nível de lactato sanguíneo e a frequência cardíaca em atletas de muay thai em fase de pré-competição. 


\section{MATERIAL E MÉTODOS}

O grupo avaliado era composto por seis indivíduos do sexo masculino, todos praticantes de Muay-Thai há pelo menos dez anos, com a idade média de $26 \pm 3,7$ anos, peso de $76 \pm 12,3 \mathrm{~kg}$, estatura $170 \pm 9 \mathrm{~cm}$. Todos os indivíduos foram previamente informados sobre os procedimentos experimentais e concordaram com a participação. $\mathrm{O}$ protocolo experimental foi aprovado pelo Comitê de Ética em Pesquisa da Pontifícia Universidade Católica do Paraná (CEP n. 1379).

Primeiramente coletou-se o lactato em repouso e anotou-se a frequência cardíaca. Em seguida, foi feito um aquecimento de cinco minutos, para evitar contusões nos atletas voluntários.

A avaliação foi realizada baseando-se nas atividades desenvolvidas em aula. A duração desse estágio foi de cinco minutos, no limite máximo de esforço, o que equivale a uma rodada (round). Imediatamente depois, coletou-se o sangue obedecendo-se os intervalos de 0,1, 3, 5, 7 min para cada indivíduo. Para a avaliação da frequência cardíaca também foram respeitados esses intervalos, utilizandose um frequencímetro $(8,20,21)$. As avaliações foram feitas duas vezes ao mês, no período de cinco meses. As coletas de sangue foram realizadas por meio de punção no lóbulo da orelha, previamente higienizada com álcool iodado. Utilizando-se uma lanceta descartável, coletou-seuma gota de sangue $(25 \mu \mathrm{L})$, que foi colocada numa fita reativa para análise da concentração de lactato $(10,21,22,23)$. Foi utilizado um analisador de lactato portátil (modelo Accutrend, Roche ${ }^{\circledR}$ ), com capacidade de detecção entre 0,8 a $22 \mathrm{mM}$, em 60 segundos (8). A frequência cardíaca foi monitorada por um frequencímetro (Polar-S120). As lancetas, as fitas de lactato e o restante dos materiais infectocontagiosos foram separados em caixas especiais e destinados ao lixo hospitalar.

Os dados experimentais foram descritos como a média dos valores \pm desvio padrão das nove amostras independentes. O coeficiente de variação de Pearson (C.V.) foi utilizado para verificar a homogeneidade de cada grupo de dados. A base estatística foi desenvolvida com o uso da análise de variância (ANOVA), concomitante ao teste Tukey para comparação das médias, com nível de significância para $\mathrm{p}<0,05$ (24).

\section{RESULTADOS E DISCUSSÃO}

$\mathrm{Na}$ Tabela 1 são apresentados os resultados do lactato no repouso. Esses dados, estatisticamente significativos, demonstram a variação do lactato sanguíneo em comparação com o repouso. Observase que o nível de lactato ao fim dos cinco minutos do round $(\mathrm{t}=0)$ aumenta cerca de duas vezes em relação ao repouso, chegando a cerca de três vezes em $t=$ 5. Parece que, nestas condições, este fato poderia prejudicar o desempenho na sequência da luta.

Ao tomar como referência o trabalho de Silva, Elifio e Pereira (25), que avaliaram atletas de cabo de guerra, exercício predominantemente anaeróbio, a média de lactato no repouso era de 1,64 $\mathrm{mM}$, diferindo dos atletas de muay thai, cujos valores médios do repouso apresentaram-se mais altos, 2,41 $\mathrm{mM}$. O atleta 5, de alta performance, apresentou o valor de 3,45 mM. Este fato deve-se, provavelmente, a uma contusão que o deixou afastado dos treinos por quatro meses. No gráfico 1, deve ser considerado que o "repouso" equivale ao tempo "zero" e após

TABELA 1 - Média \pm desvio padrão de nove experimentos independentes, do lactato sanguíneo no repouso, logo após o exercício (0 min) e nos intervalos de 1, 3, 5, 7 min após o exercício

\begin{tabular}{cccccccc}
\hline $\begin{array}{c}\text { TEMPO } \\
(\mathbf{m i n})\end{array}$ & $\begin{array}{c}\text { Média geral } \\
(\mathbf{m M})\end{array}$ & $\begin{array}{c}\text { Atl. 1 } \\
(\mathbf{m M})\end{array}$ & $\begin{array}{c}\text { Atl. 2 } \\
(\mathbf{m M})\end{array}$ & $\begin{array}{c}\text { Atl. 3 } \\
(\mathbf{m M})\end{array}$ & $\begin{array}{c}\text { Atl. 4 } \\
(\mathbf{m M})\end{array}$ & $\begin{array}{c}\text { Atl. 5 } \\
(\mathbf{m M})\end{array}$ & $\begin{array}{c}\text { Atl. 6 } \\
(\mathbf{m M})\end{array}$ \\
\hline repouso & $2,41 \pm 0,7$ & $2,47 \pm 0,7$ & $2,12 \pm 0,7$ & $2,1 \pm 0,2$ & $2,31 \pm 0,5$ & $3,45 \pm 0,6$ & $2,5 \pm 0,9$ \\
$\mathbf{0}$ & $5,34 \pm 2,0$ & $5,32 \pm 1,5$ & $4,77 \pm 2,5$ & $4,7 \pm 1,5$ & $6,55 \pm 2,2$ & $3,9 \pm 0,4$ & $5,25 \pm 2,6$ \\
$\mathbf{1}$ & $6,62 \pm 2,3$ & $6,55 \pm 2,2$ & $5,68 \pm 1,9$ & $5,82 \pm 1,9$ & $8,28 \pm 2,3$ & $5,35 \pm 0,07$ & $7,12 \pm 4,1$ \\
$\mathbf{3}$ & $6,59 \pm 2,1$ & $7,1 \pm 2,2$ & $6,25 \pm 1,9$ & $6,12 \pm 1,7$ & $7,27 \pm 1,2$ & $5,15 \pm 1,06$ & $7,57 \pm 3,9$ \\
$\mathbf{5}$ & $6,72 \pm 2,1$ & $7,26 \pm 2,1$ & $6,1 \pm 1,7$ & $6,03 \pm 1,9$ & $7,61 \pm 1,8$ & $4,7 \pm 0,2$ & $7,65 \pm 3,8$ \\
$\mathbf{7}$ & $5,77 \pm 1,3$ & $6,66 \pm 1,1$ & $5,35 \pm 0,8$ & $5 \pm 1,7$ & $6,2 \pm 1,6$ & $5,5 \pm 0,1$ & $4,7 \pm 0,1$ \\
\hline
\end{tabular}

Fonte: Elaboração dos autores. 
cinco minutos do round $\mathrm{t}=0$ iniciou-se a medida do lactato. Em relação ao lactato geral, o pico (6,72 \pm 2,1 $\mathrm{mM}$ ) ocorreu aos $5 \mathrm{~min}$, iniciando sua queda no tempo de $7 \mathrm{~min}(5,77 \mathrm{mM})$ (Tabela 1 e Gráfico 1).

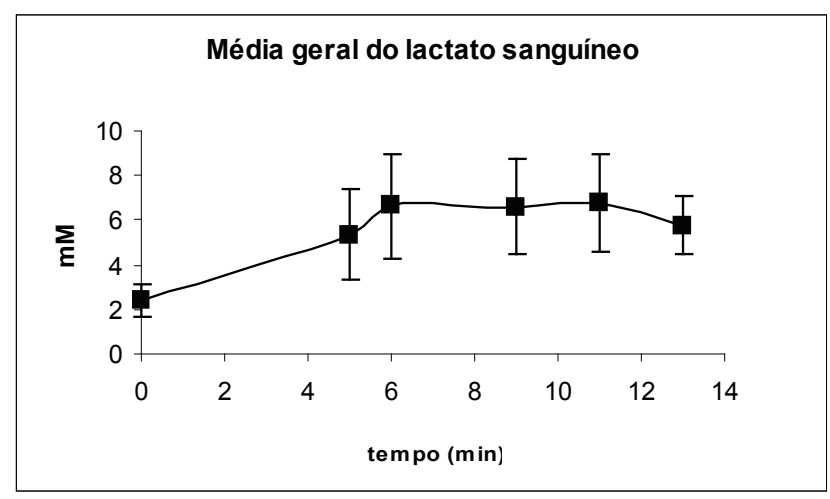

GRÁFICO 1 - Média geral \pm desvio padrão do lactato sanguíneo em relação com o tempo (min) e lactato $\mathrm{mM}$

Fonte: Elaboração dos autores.

Este aumento está de acordo com os resultados de Drigo et al. (14), que avaliaram o lactato sanguíneo em lutas de projeção e de solo em lutadores de judô, em que a média do pico de lactato foi de 6,7 $\mathrm{mM}$ em lutas de projeção e $6,5 \mathrm{mM}$ em lutas de solo. No caso de Rosa et al. (1), os resultados demonstraram que em atletas de caratê o pico de lactato médio foi de $6,3 \mathrm{mM}$, o que vem ao encontro dos resultados dos atletas de muay thai. Franchini et al. (13) observaram que judocas do sexo feminino obtiveram valores maiores no pico de lactato $(8,7 \mathrm{a} 11 \mathrm{mM})$ com o teste Wingate, $10,5 \mathrm{mM}$ a 11,5 mM nos treinos específicos e 9,9 mM e 10,8 mM no teste de simulação de luta. Este fato foi também observado em outro estudo realizado com judocas em situação de luta da classe juvenil-A, Junior e Sênior: os valores médios do pico de lactato após uma luta foram de $9,0 \mathrm{mM} ; 9,7 \mathrm{mM}$; 9,7 $\mathrm{mM}$ respectivamente (26). Comparativamente, Maceno et al. (27) avaliaram lutadores de vale-tudo, no repouso e nos intervalos de tempo de 1, 3, 5 e 7 min pós-exercício $(2,2 ; 4,54 ; 6,37 ; 4,16 ; 3,6 \mathrm{mM})$. Observa-se que o lactato máximo é semelhante aos dados de muay thai.

Em outra comparação, Pereira et al. (28) avaliaram os dados de profissionais de dança em intervalos de tempo semelhantes $(2 ; 2,8 ; 3,1 ; 3,3 \mathrm{mM})$, observando valores abaixo quando referenciados aos dados dos lutadores. Essas considerações sugerem que a atividade, o treinamento e a carga de exercício, entre outros fatores, influenciam a semelhança ou diferença nos valores do lactato. Além disso, Lima et al. (12) analisaram a correlação entre velocidade de reação motora e o lactato sanguíneo em diferentes tempos de lutas em judô. Em esforços de alta intensidade a concentração de lactato tende a ser elevada, independente da duração do combate, o que leva o atleta à fadiga, influenciando negativamente sua capacidade de acerto ao reagir a um estímulo. De modo geral, o desenvolvimento da capacidade anaeróbia láctica é essencial para que na luta os judocas suportem altas concentrações de lactato no sangue, e nos intervalos tenham capacidade de reabsorver o lactato acumulado e entrem para as lutas subsequentes capazes de reagir com maior frequência de acertos $(14,13)$. Ainda de acordo com Drigo et al. (14), o grande predomínio do metabolismo anaeróbio lático durante os treinamentos pode provocar alterações metabólicas prejudiciais ao atleta, como diminuir o rendimento aeróbio e anaeróbio alático e elevar a capacidade de reprodução de lactato. Os atletas que apresentam elevada capacidade aeróbia tendem a ter menor valor de lactato sanguíneo em qualquer tipo de atividade. Como os dados obtidos neste trabalho são semelhantes aos resultados do judô, é razoável inferir que essas considerações corroboram com nossos dados.

Os dados da frequência cardíaca (FC) são mostrados na Tabela 2, onde os indivíduos apresentaram uma frequência máxima de 173,84 bat/min, chegando próximo a $90 \%$ de sua capacidade de $\mathrm{FC}_{\text {máx }}$.

No Gráfico 2 observa-se também a variação da frequência cardíaca, mostrando que o protocolo utilizado elevou consideravelmente a frequência cardíaca dos atletas. Neste gráfico também deve ser considerado que o "repouso" equivale ao tempo "zero" e após 5 minutos do round $(\mathrm{t}=0)$ iniciou-se a medida da FC.

A frequência cardíaca observada entre os tempos $\mathrm{t}=0 \mathrm{e} \mathrm{t}=1$ caiu aproximadamente $35 \%$ do pico máximo; este fato, que simula um intervalo de luta, parece demonstrar uma recuperação satisfatória para os atletas em combate.

Sampaio e Velozo (7) inferem que, quanto melhor a condição física do indivíduo, mais baixa tenderá a ser sua frequência cardíaca em repouso. Uma característica de atletas é uma frequência cardíaca lenta associada a um volume de ejeção relativamente grande, indicando um sistema circulatório bastante eficiente. Carvalho etal. (6) observaram em triatletas, a partir de testes com bicicleta ergométrica e ciclossimulador, os resultados da frequência cardíaca máxima nos valores de 180 e 182 bat/min 
TABELA 2 - Média e desvio padrão da frequência cardíaca, no repouso e logo após 5 min de exercício

\begin{tabular}{cccccccc}
\hline $\begin{array}{c}\text { TEMPO } \\
\text { (min) }\end{array}$ & $\begin{array}{c}\text { Média geral } \\
\text { FC (bat/min) }\end{array}$ & Atl. 1 & Atl. 2 & Atl. 3 & Atl. 4 & Atl. 5 & Atl. 6 \\
\hline Repouso & $65,48 \pm 8,8$ & $65,33 \pm 10,3$ & $61,33 \pm 8,8$ & $63,12 \pm 57,8$ & $64,85 \pm 5,1$ & $84 \pm 7$ & $71,75 \pm 7,8$ \\
$\mathbf{0}$ & $173,89 \pm 10,1$ & $174,11 \pm 6,3$ & $173,77 \pm 13,2$ & $174,87 \pm 6,2$ & $172,57 \pm 12,4$ & $161 \pm 21,2$ & $180,5 \pm 3,4$ \\
$\mathbf{1}$ & $114,07 \pm 11,8$ & $116,33 \pm 9,2$ & $114,44 \pm 9,5$ & $120,75 \pm 15$ & $103,28 \pm 3,4$ & $99,5 \pm 2,1$ & $121 \pm 11,5$ \\
$\mathbf{3}$ & $97,66 \pm 9,4$ & $103,33 \pm 10,5$ & $93,44 \pm 8,2$ & $100 \pm 2,8$ & $88,85 \pm 5,0$ & $95 \pm 5,6$ & $106,5 \pm 10,6$ \\
$\mathbf{5}$ & $95,02 \pm 7,7$ & $98,22 \pm 4,6$ & $92,22 \pm 5,4$ & $97,62 \pm 4,7$ & $86,28 \pm 5,1$ & $100,5 \pm 3,5$ & $101,5 \pm 13,4$ \\
$\mathbf{7}$ & $93,1 \pm 8,3$ & $97,66 \pm 3,6$ & $89,33 \pm 9$ & $93 \pm 6,6$ & $87,14 \pm 3,5$ & $94,5 \pm 2,1$ & $101,25 \pm 14,7$ \\
\hline
\end{tabular}

Fonte: Elaboração dos autores.

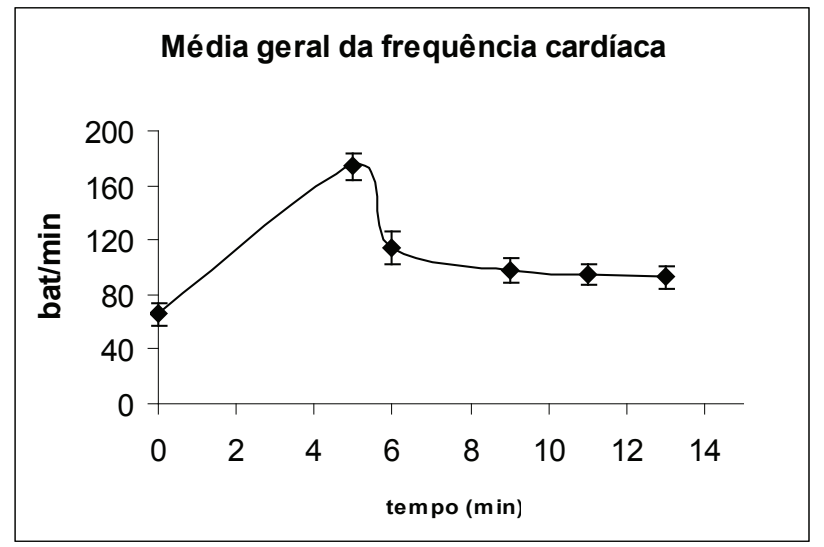

GRÁFICO 2 - Média geral \pm desvio padrão da frequência cardíaca no repouso e intervalos de tempo de $0,1,3,5,7 \mathrm{~min}$

Fonte: Elaboração dos autores.

respectivamente; assim como Milanez et al. (29) observaram em seus experimentos com caratecas a frequência cardíaca máxima de 194 bat/min. Esses resultados vêm ao encontro dos dados obtidos nos atletas de muay thai que apresentaram valores semelhantes. Fato que não é restrito aos praticantes de esportes de contato (30): Silva et al. (4) relatam que a frequência cardíaca em repouso (70 bat/ min) e após exercício (194 bat/min) em bailarinos não difere a priori dos dados, neste parâmetro, dos lutadores de judô e muay thai.

No Gráfico 3 foi utilizada a porcentagem como medida de comparação entre os fatores lactato sanguíneo e frequência cardíaca. Observase neste gráfico que estes fatores são inversamente proporcionais, ou seja, no quinto minuto, logo após o exercício, a frequência cardíaca encontra-se no seu estado máximo; em contrapartida, olactato sanguíneo ainda esta crescente. Com o decorrer do primeiro intervalo de tempo, o lactato começa a se elevar e a frequência a decair abruptamente. Nos outros intervalos de tempo observa-se um declínio mais leve da frequência cardíaca e, consequentemente, um aumento da concentração do lactato sanguíneo no sangue. Este aumento se dará até o quinto minuto após o exercício, visto que no $13^{\circ}$ minuto de todo o experimento o lactato começa a decair levemente.

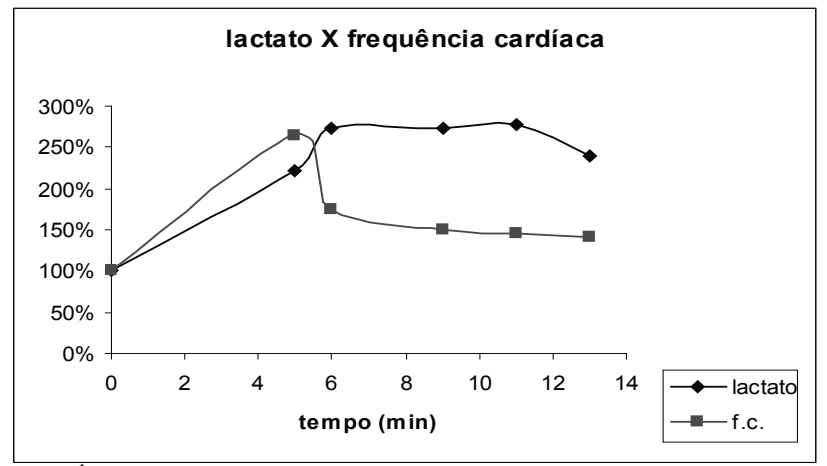

GRÁFICO 3 - Percentual comparativo entre o lactato sanguíneo versus frequência cardíaca

Fonte: Elaboração dos autores.

\section{CONCLUSÃO}

Os atletas de lutas marciais, com referência aos valores de lactato apresentaram números semelhantes aos do judô. Os dados obtidos aqui podem fornecer subsídios para reavaliações de protocolos de treinamento para os atletas de muay thai, para que eles atinjam, no período de competição, um nível de recuperação lática mais efetivo, aumentando assim sua performance em lutas. Com relação à frequência cardíaca os valores parecem mostrar uma adaptação ao treinamento proposto. Entretanto, os resultados possuem diferenças individuais, as quais devem ser analisadas com cautela. Neste protocolo experimental se demonstrou um 
indicador importante no condicionamento físico dos atletas de muay thai, contudo, sugere-se a inserção de mais rounds, para avaliar outros parâmetros de aptidão física.

\section{AGRADECIMENTOS}

Agradecemos ao Programa Institucional de Bolsas de Iniciação Científica PUCPR/PIBIC pelo suporte financeiro.

\section{REFERÊNCIAS}

1. Rosa, MW, Vale, CK, Redondo, CM, Junges RD, Zanoni JHB. Análise cinética do lactato durante uma aula de Karatê - Dô shotokan. RBPFEX. 2007;1(6):39-44.

2. Foss LM, Keteyian JS. Bases fisiológicas do exercício e do esporte. 6a ed. Rio de Janeiro: Guanabara koogan; 2000.

3. Ramos RS, Lopes ES, Leonel L, Rocha R, Matsushigue KA, Gobatto CA. Treinamento aeróbio em bailarinas: influência sobre a realização de coreografias de 4 e 8 minutos de duração. Rev Paul Educ Fís. 1995;9(1):26-36.

4. Silva PRS, Romano A, Gava NS, Dourado MP, Yazbeck P, Shinzato GT, et al. Perfil de aptidão cardiorrespiratória e metabólica em bailarinos profissionais. Rev Brás Med Esporte. 1999;5(1):81-5.

5. Weineck J. Biologia do esporte. São Paulo: Manole; 2000. p. 210-65.

6. Carvalho Jr. ES, Santos ALG, Schneider AP, Beretta L, Tebexreni AS, Cesar MC, et al. Análise comparativa da aptidão cardiorrespiratória de triatletas, avaliados em ciclossimulador e bicicleta ergométrica. Rev Bras Ciên e Mov. 2000;8(3):21-4.

7. Sampaio E, Velozo E. Fisiologia do esforço. Ponta Grossa: Ed da UEPG; 2001.

8. Silva PRS, Inarra LA, Vidal JRR, Oberg ARB, Fonseca A; Roxo CDN, et al. Níveis de lactato sanguíneo, em futebolistas profissionais, verificados após o primeiro e o segundo tempos em partidas de futebol. Acta Fisiátrica. 2000;7(2):68-74.
9. Arias PM, Diaz PD, Aristizabal JC, Jaramilo NH. Efeitos da desidratação, durante exercício sub-máximo de longa duração, na concentração sanguínea do lactato, na freqüência cardíaca e na percepção subjetiva do esforço. Rev Bras Ciên e Mov. 2001;9(4):41-6.

10. Baptista RR, Oliveira LG, Figueiredo GB, Contieri JR, Loss JF, Oliveira AR. Limiar de lactato em remadores: comparação entre dois métodos de determinação. Rev Bras Med Esporte. 2005;11(4):247-50.

11. Benetti M, Santos RT, Carvalho, T. Cinética de lactato em diferentes intensidades de exercícios e concentrações de oxigênio. Rev Bras Med Esporte. 2000;6(2): 51-6.

12. Lima EV, Tortoza C, Rosa LCL, Martins RABL. Estudo da correlação entre a velocidade de reação motora e o lactato sanguíneo, em diferentes tempos de luta de judô. Rev Bras Med Esporte. 2004;10(5):339-43.

13. Franchini E, Matsushigue KA, Kiss MAPD, Sterkowicz S. Estudo de caso das mudanças fisiológicas e de desempenho de judocas do sexo feminino em preparação para jogos Pan-Americanos. Rev Bras Ciên e Mov. 2001;9(2):21-7.

14. Drigo AJ, Amorim AR, Martins CJ, Molina R. Demanda metabólica em lutas de projeção e de solo no judô: estudo pelo lactato sanguíneo. Motriz. 1996;2(2):80-6.

15. Basset DR, Merril PW, Nagle FJ, Agre JC, Sampedro RMF. Taxa de declínio do lactato sanguíneo após exercício em bicicleta ergométrica em indivíduos treinados em endurance e em não treinados. Rev Bras Ativid Físic Saúde. 1995;1(1):9-7.

16. Denadai BS, Ruas VDA, Figueira TR. Efeito da cadência de pedalada sobre as respostas metabólica e cardiovascular durante o exercício incremental e de carga constante em indivíduos ativos. Rev Bras Med Esporte. 2005;11(5):286-90.

17. Donovan CM, Brooks GA. Endurance training affects lactate clearance, not lactate production. Am J Physiol. 1983;244(1):83-92.

18. Mcardle WD, Kacth FI, Kacth LV. Fisiologia do exercício - energia, nutrição e desempenho humano. 4a ed. Rio de Janeiro: Guanabara Koogan; 2003. 
19. Alonso DO, Forjaz CM, Rezende LO, Braga AMFW, Barretto, ACP, Negrão CE, et al. Comportamento da freqüência cardíaca e da sua variabilidade durante as diferentes fases do exercício físico progressivo Máximo. Arq Bras Cardiol. 1998;71(6):787-92.

20. Lima Silva AE, Gagliardi JFL, Lotufo, RFM, Kiss MAPDM. Ajustes lineares vs quadráticos da curva de consumo de oxigênio em teste progressivo. Rev Bras Ciênc Mov. 2003;11(4):13-8.

21. Niess AM, Fehrenbach E, Strobel G, Roecker K. Individual differences in self-reporter heat tolerance. Is there a link to the cardio circulatory, thermoregulatory and hormonal response to endurance exercise in heat? Medicine \& Science in Sport \& Exercise. 2003;43(1):386-92.

22. Denadai B, Figueira TR, Favaro O, Gonçalvez M. Effect of the aerobic capacity on the validity of the anaerobic threshold for determination of the maximal lactate steady state em cycling. Braz J Med Biol Res. 2004;37(10):1551-6.

23. Niess AM, Fehrenbach E, Strobel G, Roecker K. Evaluation of stress responses to interval training at low and moderate altitudes. Medicine \& Science in Sport \& Exercise. 2002; 1:263-269.

24. Magnusson W, Mourão G. Estatística sem matemática: a ligação entre as questões e a análise. Londrina: Planta; 2003.

25. Silva FP, Elífio SL, Pereira LF. Avaliação do nível de lactato sanguíneo em praticantes de exercícios isométricos. Estud Biol. 200527(60):19-24.

26. Franchini E, Takito MY, Lima JRP, Haddad S, Kiss MAP, Regazzini M, et al. Características fisiológicas em testes laboratoriais e resposta da concentração de lactato sanguíneo em três lutas em judocas das classes Juvenil-A, Junior e Sênior. Rev Paul Educ Fís. 1998;12(1):5-16.

27. Maceno T, Zachow MS, Ribeiro FL, Silva FP, Elífio ES, Pereira LF. Avaliação do lactato sanguíneo em lutadores profissionais. Anais do $28^{\circ}$ Simpósio Internacional de Ciência do Esporte. 2005;13(4): 178.

28. Pereira LF, Silva FP, Maceno T, Zachow MS, Quadrado ET, Ribeiro FP, et al. Avaliação do lactato sanguíneo em bailarinos profissionais. Anais do $28^{\circ}$ Simpósio internacional de Ciência do Esporte. São Paulo: Simpósio Internacional de Ciência do Esporte; 2005.
29. Milanez VF, Santos KE, Gonçalvez CGS, Lima MCS, Balikian P, Ribeiro LFP. Comparação entre protocolo contínuo e intermitente para determinação do consumo máximo de oxigênio em Karatecas. Anais do $28^{\circ}$ Simpósio Internacional de Ciência do Esporte. São Paulo: Simpósio internacional de Ciência do esporte; 2005.

30. Souza TNT, Yamaguti SAL, Campbell CSG, Simões HG. Identificação do lactato mínimo e glicose mínima em indivíduos fisicamente ativos. Rev Bras Ciênc Mov. 2003;11(2):71-75.

Recebido: 13/08/2008

Received: 08/13/2008

Aprovado: $14 / 10 / 2008$

Approved: 10/14/2008 